\title{
Color Doppler Evaluation of Isovolumetric Relaxation Time and of Signals Arising from Axons of the Median Nerve as a Means to Evaluate Mitochondrial Functionality in the Context of Immunotherapy of Cancer and Chronic Conditions Associated with Mitochondrial Dysfunction
}

\author{
Stefania Pacini and Marco Ruggiero \\ Silver Spring Sagl, Arzo-Mendrisio, Switzerland
}

Article history

Received: 11-09-2019

Revised: 01-10-2019

Accepted: 11-10-2019

Corresponding Author:

Stefania Pacini

Silver Spring Sagl,

Via Raimondo Rossi 24, Arzo-

Mendrisio 6864, Switzerland

Email: info@bravo-europe.com

\begin{abstract}
Reactive Oxygen Species (ROS) arising from the disruption of mitochondrial respiration act as endogenous mutagens and tumor promoters. When production of ROS exceeds the capacity of DNA repair mechanisms, random mutations and aneuploidy ensue, thousands of genes become unbalanced and genetic/epigenetic chain reactions lead to progressive aneuploidy with only two outcomes: karyotypes so altered that are not viable or karyotypes of autonomous, immortal, cancer cells. Consistent with the concept that abnormalities of cellular respiration in mitochondria precede DNA alterations in the nucleus, transplant of normal mitochondria suppresses tumorigenesis and metastases in vitro and in vivo. However, the transplant of mitochondria is an experimental procedure that cannot be easily applied to clinical practice. In order to overcome this limitation, we designed a novel formula based on microbial chondroitin sulfate, vitamin $\mathrm{D}_{3}$ and ultrapure phosphatidylcholine, molecules that are known to restore mitochondrial functionality and suppress ROS production. Here we describe how such an approach can be evaluated by color Doppler ultrasonography of the radial artery and measure of the Isovolumetric Relaxation Time (IVRT). In addition, we show for the first time color Doppler signals originating from axons of the median nerve that may be indicative of quantum phenomena at the level of mitochondria. We propose the use of the original ultrasonographic techniques here described for evaluating the effectiveness of substances or strategies aimed at restoring mitochondrial functionality at the macroscopic and quantum levels.
\end{abstract}

Keywords: Cancer, Mitochondria, Reactive Oxygen Species, Chondroitin Sulfate, Vitamin D, Phosphatidylcholine, Isovolumetric Relaxation Time, Median Nerve, Quantum Entanglement

\section{Introduction}

Cancer is a complex disease where metabolic, genetic and epigenetic factors are closely intertwined and produce such a variety of aberrations in all aspects of cellular physiology that is difficult to ascertain which is the cause and which is the effect. According to decadeold theories that have received experimental confirmation in the past few years, initiation of cancer can be found in metabolic alterations at the level of mitochondria with consequent production of Reactive Oxygen Species (ROS) that act as endogenous mutagens and tumor promoters (Seyfried, 2015). When prolonged mitochondrial damage is of such an extent that production of ROS exceeds the capacity of DNA repair mechanisms, random mutations occur with the risk of inducing aneuploidy, a condition that leads to the imbalance of thousands of genes at a time. Such an occurrence leads to genetic and epigenetic chain reactions that either lead to non-viable karyotypes, or in relatively rare cases that develop over time, to immortal cancer cells in a process that resembles speciation (Hirpara et al., 2018). Chemicals or physical treatments such as ionizing radiations, that are defined mutagens for 
their ability to interact with DNA, are also known disruptors of mitochondrial respiration; defective mitochondrial respiration associated with compensatory fermentation is then responsible for the deluge of mutations that culminate in aneuploidy and, ultimately, in speciation (Seyfried, 2015; Hirpara et al., 2018). Pervasive genetic and epigenetic instability culminating in aneuploidy and speciation further disrupts mitochondrial respiration up to the point that the process becomes autonomous and self-sustaining. In addition, disruption of mitochondrial respiration by carcinogens with consequent compensatory fermentation leads to tissue acidification that, in turn, destabilizes the cancer microenvironment and favors local cancer growth as well as metastatization, processes that are further sustained by acidification- induced neo-angiogenesis.

If the primum movens of cancer lays in alterations of mitochondrial functionality rather than in mutations of nuclear DNA in somatic cells, it may be argued that replacing defective mitochondria with functional ones should prevent or neutralize tumorigenesis. This concept is consistent with the decade-old theory of Nobel Laureate Otto Warburg postulating that respiratory insufficiency is at the origin of cancer (Warburg, 1956). Plenty of evidence gathered in the past 30 years demonstrates that transplant of normal mitochondriaoften together with normal cytoplasm-suppresses tumorigenesis in vitro and in vivo (Seyfried, 2015). Normal mitochondria, by restoring cellular respiration would restore normal energy homeostasis, thus leading to cancer cell differentiation. In this context, transplant of normal mitochondria would be effective even in advanced stages of tumor progression and also in the presence of aneuploidy as demonstrated by studies describing how normal mitochondria inhibit tumorigenicity even in the presence of extensive mutations in the nucleus of cancer cells (Seyfried, 2015).

As far as clinical applications are concerned, mitochondrial transplant has recently been considered a promising approach for mitochondrial diseases and cancer (McCully et al., 2016; Chang et al., 2019) and mitochondrial transfer has been patented under the name "Autologous Germline Mitochondrial Energy Transfer" for human assisted reproduction (Woods and Tilly, 2015). However, it is difficult to conceive the application of this type of protocols to conditions that are characterized by mitochondrial dysfunction such as cancer, neurodegenerative diseases, autism or aging and we are not aware of studies reporting successful application of mitochondrial transplant in these conditions. A recent study performed on human breast cancer cells in vitro demonstrated that the anti-cancer effects of mitochondrial transplant are associated with significantly decreased ROS production and shift in energy production with less energy deriving from glycolysis (Chang et al., 2019). Transplant of normal mitochondria into human breast cancer cells, in addition to direct anti-cancer effects, also increased sensitivity to known chemotherapeutic drugs such as Doxorubicin and Paclitaxel (Chang et al., 2019). Since these drugs have different mechanisms of action-Doxorubicin inhibits transcription and replication of nuclear DNA and increases DNA damage by inhibiting topoisomerase II; Paclitaxel is a cytoskeletal drug that inhibits mitosis by stabilizing microtubules-it may be hypothesized that restoring mitochondrial activity in cancer cells not only induces cancer cell apoptosis per se but also makes cancer cells more sensitive to different, possibly all, types of chemotherapy.

Based on the studies and considerations mentioned above, we developed a formula that has the goal of restoring mitochondrial function without the need of a mitochondrial transplant. Such an approach is based on combining the effects of known regulators and restorers of mitochondrial function, the glycosaminoglycan chondroitin sulfate, vitamin $\mathrm{D}_{3}$ and a major constituent of mitochondrial membrane, the phospholipid phosphatidylcholine. This approach and its constituents have been thoroughly described in two recent papers (Ruggiero and Pacini, 2018a; 2018b) and clinical results associated with this approach have also been recently described (Antonucci et al., 2018; 2019a; 2019b). Here, we propose a rapid and simple method to assess the efficacy of such an approach in modulating energy production at the level of mitochondria. This method is based on the evaluation of Isovolumetric Relaxation Time (IVRT) by color Doppler ultrasonography of the radial artery. We also discuss the implications arising from the original observation of Doppler signals originating from within the axons of the median nerve.

\section{Materials and Methods}

IVRT, often designated also isovolumic relaxation time, is used in cardiology as an indicator of diastolic physiology or dysfunction. It is the time comprised between the closure of the aortic valve and the opening of the mitral valve. During this time, the fibers of the myocardium relax while the volume of the left ventricle remains constant until the opening of the mitral valve. The length of IVRT is dependent on the amount of energy generated by mitochondria; during this interval, cardiomyocytes extrude calcium and this is achieved by using all the energy, under the form of ATP, generated by mitochondria. Therefore, in the presence of defective energy production at the level of mitochondria, IVRT is longer; on the other hand, substances or treatments that increase energy production at the level of mitochondria decrease IVRT. In other words, IVRT is inversely proportional to the energy generated by mitochondria in cardiomyocytes. In cardiology, IVRT is measured by combining trans-thoracic Doppler echocardiography and M-mode sonography or phonocardiogram and trans- 
mitral Doppler echocardiography. Using these techniques, it was demonstrated that normal IVRT is around $60 \mathrm{~ms}$ (Brecker et al., 1992). It is worth noticing that the evaluation of IVRT is a rather complex procedure that is usually performed in clinics or departments dedicated to cardiology. Here, we describe a simplified method to evaluate IVRT by color Doppler ultrasonography of the radial artery using commonly available generic ultrasound systems. The ultrasounds used for evaluating IVRT, also known as sub-thermal ultrasounds, are considered harmless for the human body up to the point that they have been and are being, used in obstetrics and gynecology for decades to obtain images in utero. Any modern ultrasound system with a linear probe for muscle-skeletal examination and color Doppler application can be used. The first step of the procedure consists in finding the pulse of the radial artery by palpation of the volar surface of the wrist. The second step consists in visualizing the radial artery in an axial section at the level of the wrist in B-mode; this appears as a pulsating black circle (Fig. 1).

The radial artery can be measured and studied for anomalies. Once the radial artery has been visualized, the color Doppler is turned on and the flow of blood can be visualized (Fig. 2).

At first, the probe has to be tilted towards the elbow of the subject so to have a homogeneous image of the flow of blood coming towards the probe and being recorded as "red" according to the known rule of Doppler ultrasonography "Blue Away Red Towards" (BART). At this point, the probe can be tilted towards the hand of the subject so that the flow in the radial artery appears blue and the application to measure velocities and times can be turned on (Fig. 3).

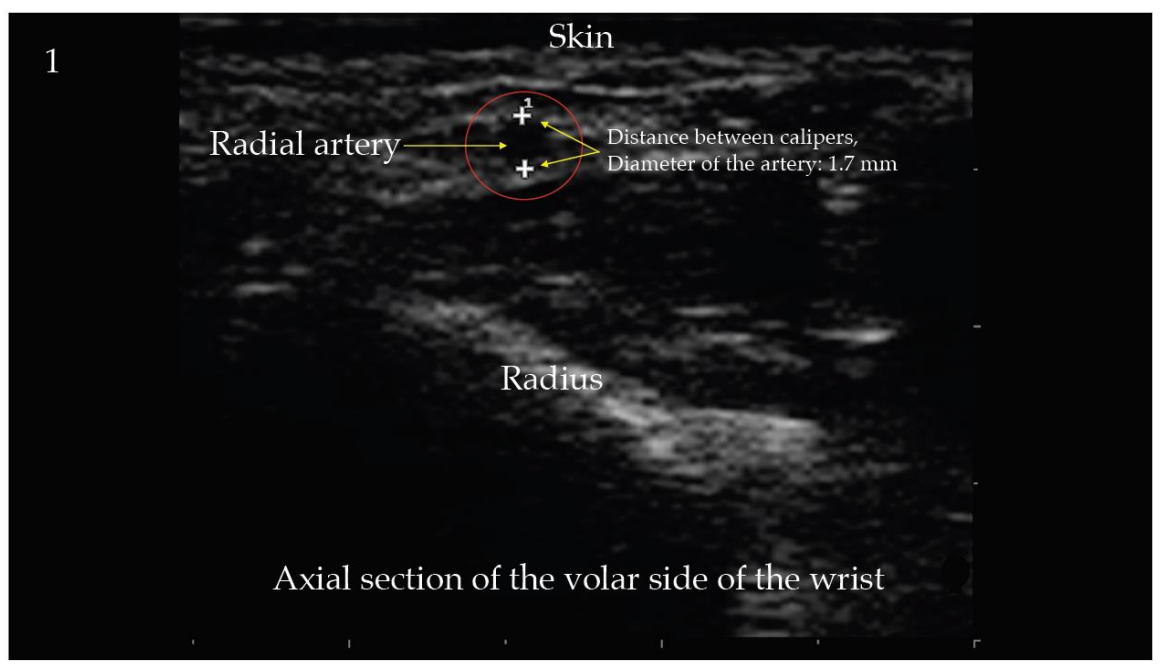

Fig. 1: Ultrasonography of the volar side of the wrist, axial section. The radial artery is visualized as a black circle and has a diameter of $1.7 \mathrm{~mm}$

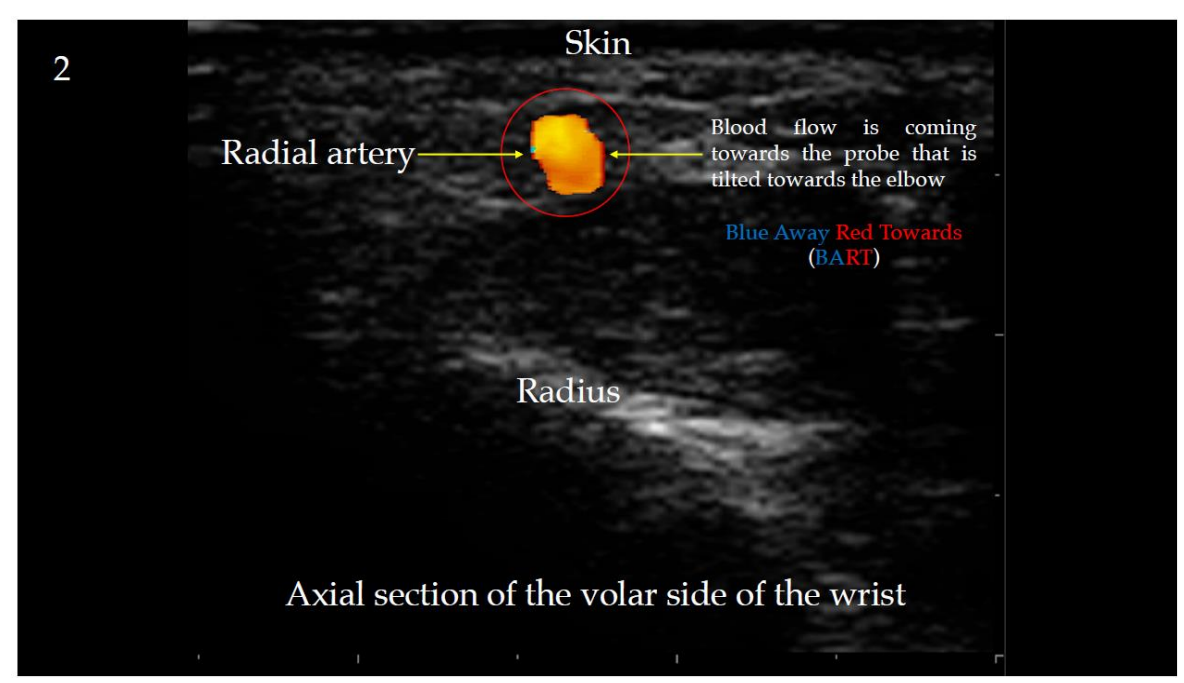

Fig. 2: Color Doppler ultrasonography of the radial artery. The probe is tilted towards the elbow of the subject 


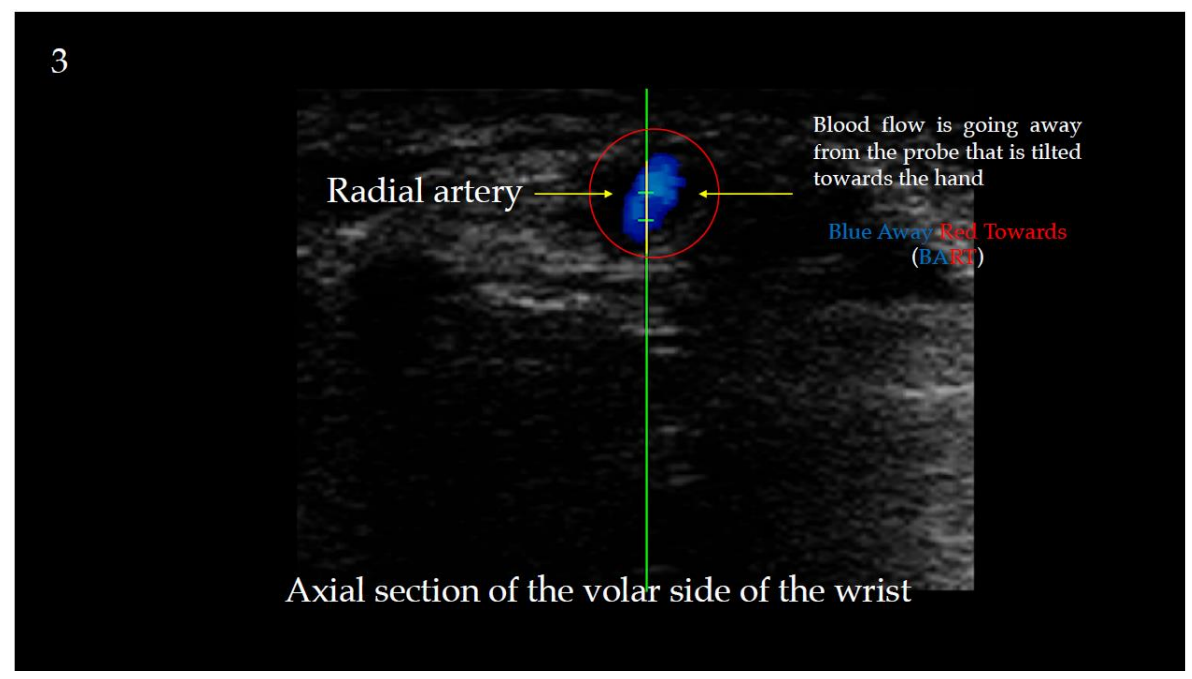

Fig. 3: Color Doppler ultrasonography of the radial artery. The probe is tilted towards the hand of the subject and the cursor to measure velocities and times is applied

\section{Results and Discussion}

According to our knowledge, this is the first description of a technique to evaluate IVRT at the level of the radial artery. The measure of IVRT by color Doppler ultrasonography of the radial artery following the procedure described above is shown in Fig. 4.

IVRT is evaluated by measuring the time interval corresponding to the negative curve following the systolic peak. Figure 4 shows an example of IVRT measured with this procedure with a resulting value of $118 \pm 3.2 \mathrm{~ms}$. This value appears longer than those measured by transthoracic ultrasonography and this difference is due to the measure at the periphery. Although this measure is indirect in comparison to that obtained by trans-thoracic ultrasonography, the procedure has the convenience that it can be performed in a few minutes, with no discomfort for the subject and no need to undress, with obvious advantages. It is worth noticing that this procedure has not the goal to diagnose diastolic dysfunction but to evaluate the effectiveness of strategies aimed at increasing mitochondrial energy production with the ultimate goal of restoring mitochondrial functionality in cancer cells as well as in conditions associated with mitochondrial dysfunction. In addition, the procedure described above allows simple and rapid visualization of the median nerve in the volar side of the forearm by sliding the probe towards the elbow and placing it at 90 degrees in order to obtain an axial image as in the example of Fig. 5.

The median nerve has an ovoidal shape and is surrounded by the epineurium that appears as a relatively thick (0.4-0.5 mm) hyper-echoic-white-linear structure. The perineurium surrounding the fascicula appears as a thinner $(\sim 0.3 \mathrm{~mm})$ hyper-echoic linear structure. After having visualized the nerve, the resulting spectral curve is shown in Fig. 6 and probe is tilted towards the elbow so to capture any flow moving towards the probe. Application of color Doppler to the inside of the median nerve and analysis of the resulting spectral curve is shown in Fig. 6 and 7.

The pattern evidenced in Fig. 7 appears peculiar and we are not aware of any description of these color Doppler signals originating from inside the median nerve. We attribute these signals to mitochondria traffickingflow-inside the axons of the nerve. It is well assessed that energy provided by mitochondria is required for synaptic function and, more in general, for all neurologic functions (Sajic et al., 2013; Nunn et al., 2016) and it has been demonstrated that mitochondria move at a speed between 0.3 and $0.7 \mu \mathrm{m} / \mathrm{s}$ in peripheral nerves (Sajic et al., 2013). Peripheral nerve stimulation increases the number and the speed of mitochondria moving towards synapses that are areas of high energy requirements. It can be therefore hypothesized that the Doppler signals observed in Fig. 7 are associated with trains of mitochondria moving inside the axons of the median nerve. Such a flow of mitochondria and the possibility to study it as proposed here for the first time, implies interesting consequences as far as cancer and other diseases associated with mitochondrial dysfunction are concerned. Evolution of mitochondria has favored the generation of energy at the so-called "redox sweet spot" (Nunn et al., 2016), where energy production with minimal ROS generation is optimized for health and longevity. Such optimization is achieved through quantum phenomena that involve electron tunneling, prolonged quantum coherence and entanglement. The strong electromagnetic fields generated by electron transport inside mitochondria (Guo et al., 2018) could be responsible for protection against decoherence and there is evidence supporting the hypothesis 
that in complex biological systems quantum coherence can be sustained for times orders of magnitude longer than those achievable in simplified quantum systems at room temperature (Nunn et al., 2016). This type of biological quantum coherence is deemed responsible for consciousness and its intrinsic role in the universe (Hameroff and Penrose, 2014). The observation that mitochondria move at measurable speeds, thus generating further, stronger, electromagnetic fields, adds a layer of complexity that may lead to an augmented state of quantum coherence. We are not aware as to whether the signals originating from the median nerve that we have captured using the original procedure described above are associated with this augmented state of quantum coherence or are macroscopic evidence of entanglement. It is tempting to speculate that the signatures of these signals correspond to a level of information that we have just begun to decipher. For example, the two signals circled in red in Fig. 7, designated with the letter "A", appear to be identical and may represent the macroscopic signature of a phenomenon of entanglement or synchronization occurring in the electron clouds of fastmoving mitochondria. Similar, albeit not identical in size, couples can be observed for the signals circled in " $\mathrm{B}$ " and "C", whereas the signals in " $\mathrm{D}$ " and " $\mathrm{E}$ " do not show repetitive patterns. It is also interesting to notice that, if the signals in "A" are associated with mitochondrial quantum entanglement, they may also be indicative of the occurrence of a wormhole; this is a structure enabling non-conventional transfer of information between separate points in time and space, independently of spatial contiguity or direction of the arrow of time as we recently discussed in the context of virus-associated information transfer (Pacini and Ruggiero, 2019). The signals evidenced for the first time in Fig. 7 may also serve to study in real-time the effects of hormesis in mitochondria and, more specifically, the effects of substances or strategies aimed at improving mitochondrial functionality. It is well assessed that mitochondria-generated energy has an important role in hormesis since mild stressors elicit an adaptive response that increases mitochondrial efficiency (Tapia, 2006). Hormesis, by favoring the evolutionary selection of more efficient electron transport chains in mitochondria would then prevent and/or counteract mitochondrial dysfunction with associated excessive ROS production. Such a concept of hormesis in the context of radiations and cancer is well documented (Luckey, 2008; Shibamoto and Nakamura, 2018) and it can be hypothesized that the effects observed in a recent study on multiple myeloma can be ascribed to phenomena of hormesis leading to enhanced mitochondrial efficiency possibly through enhancement of coherent states at quantum level (Antonucci et al., 2019b). Whatever the case, the procedures described above represent a rapid and simple method to study mitochondrial functionality at the macroscopic and possibly at the quantum level, with a number of practical applications. The rationale for suggesting implementation of the ultrasonographic procedures described here in oncology and in other conditions associated with low energy production at the level of mitochondria is the following; if a substance or a treatment is able to increase energy production at the level of cardiomyocytes and enhance quantum coherence at the level of moving mitochondria, it may also restore mitochondria functionality in other tissues including cancerous tissues. Consistent with this concept, we have previously demonstrated that immunotherapeutic agents successfully used in cancer, in particular vitamin D receptor (VDR) agonists complexed with amphipathic macromolecules and lipophilic compounds, are able to increase IVRT (Pacini et al., 2013).

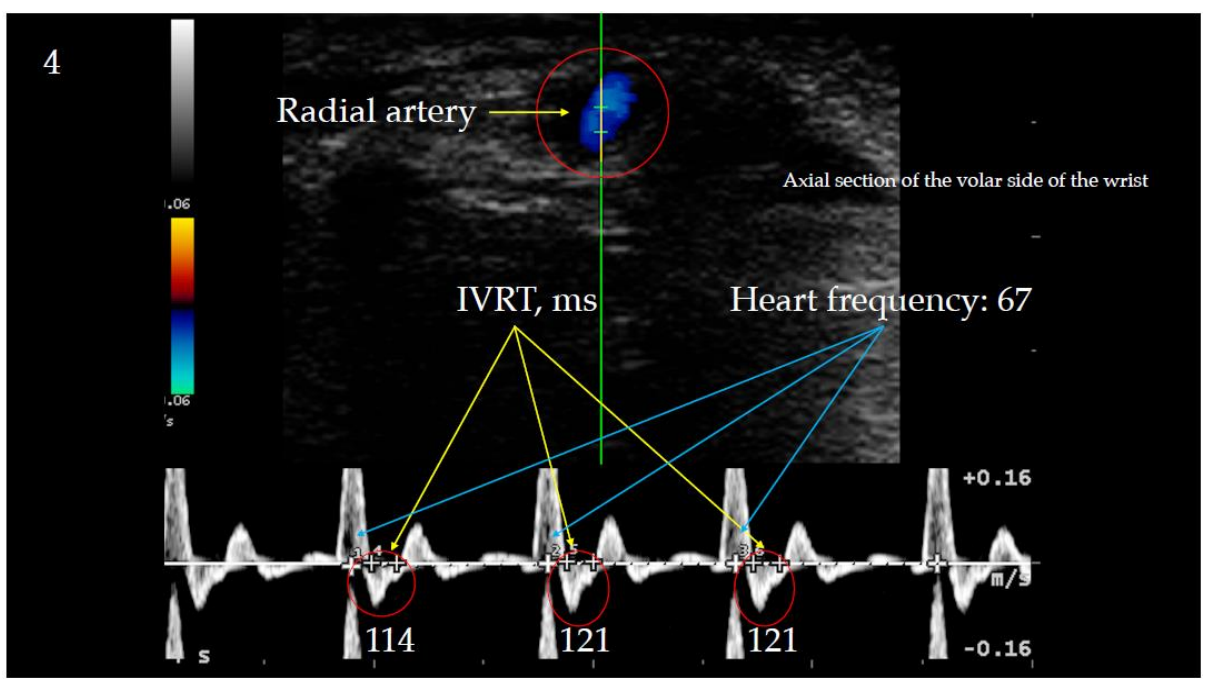

Fig. 4: Evaluation of isovolumetric relaxation times (IVRT) by color Doppler ultrasonography 


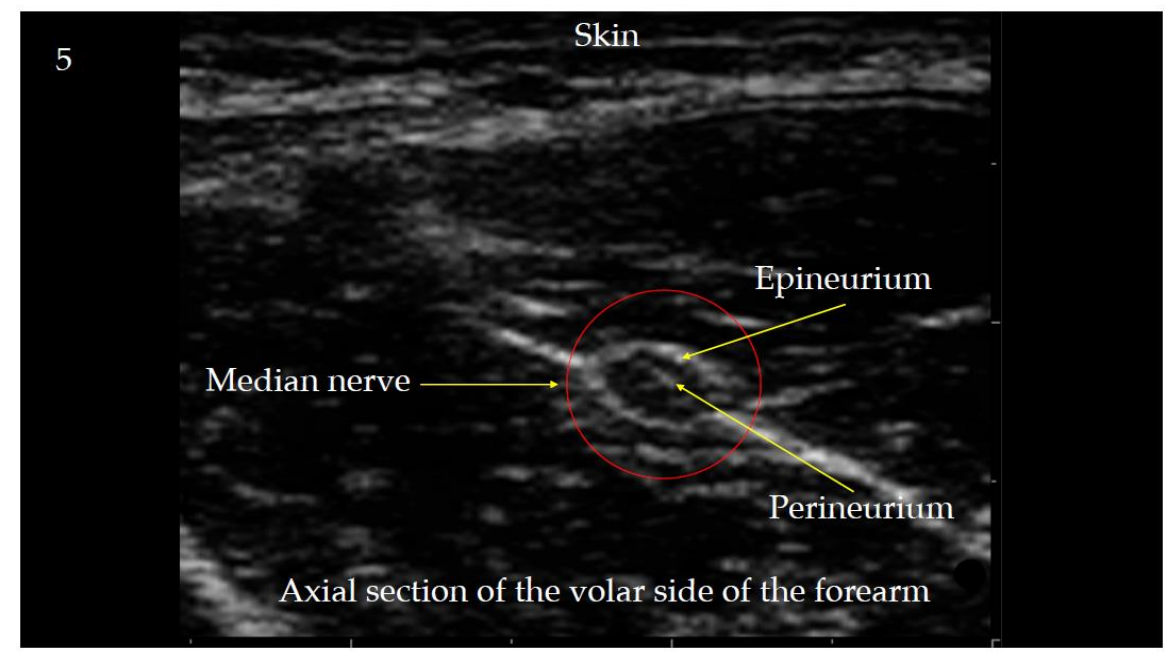

Fig. 5: Ultrasonography of the median nerve

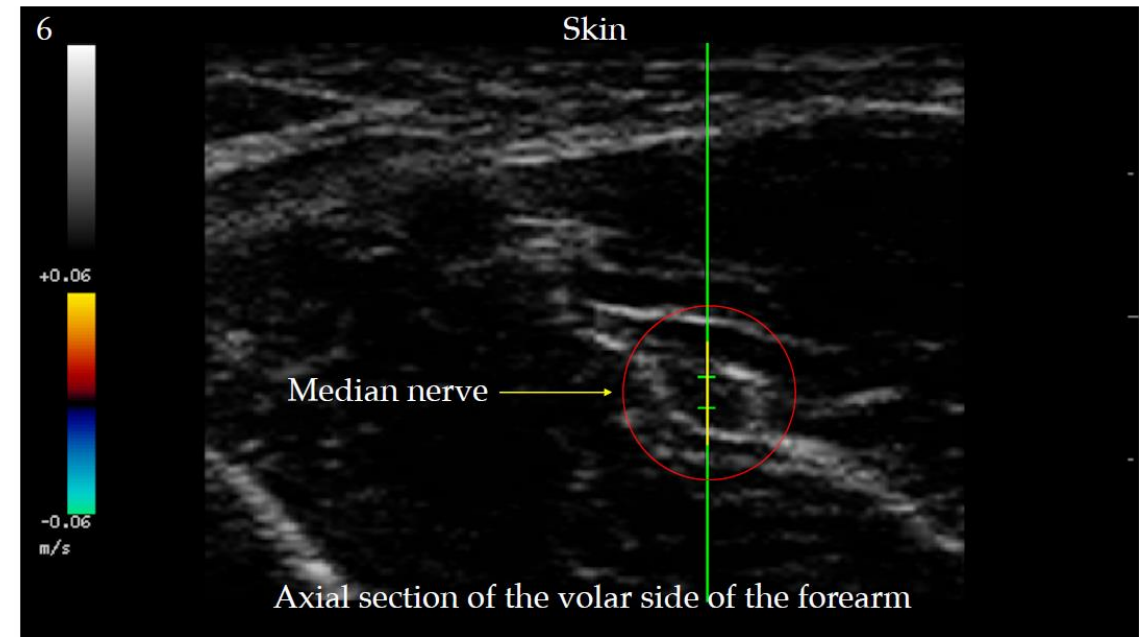

Fig. 6: Ultrasonography of the median nerve, positioning of the cursor of the color Doppler

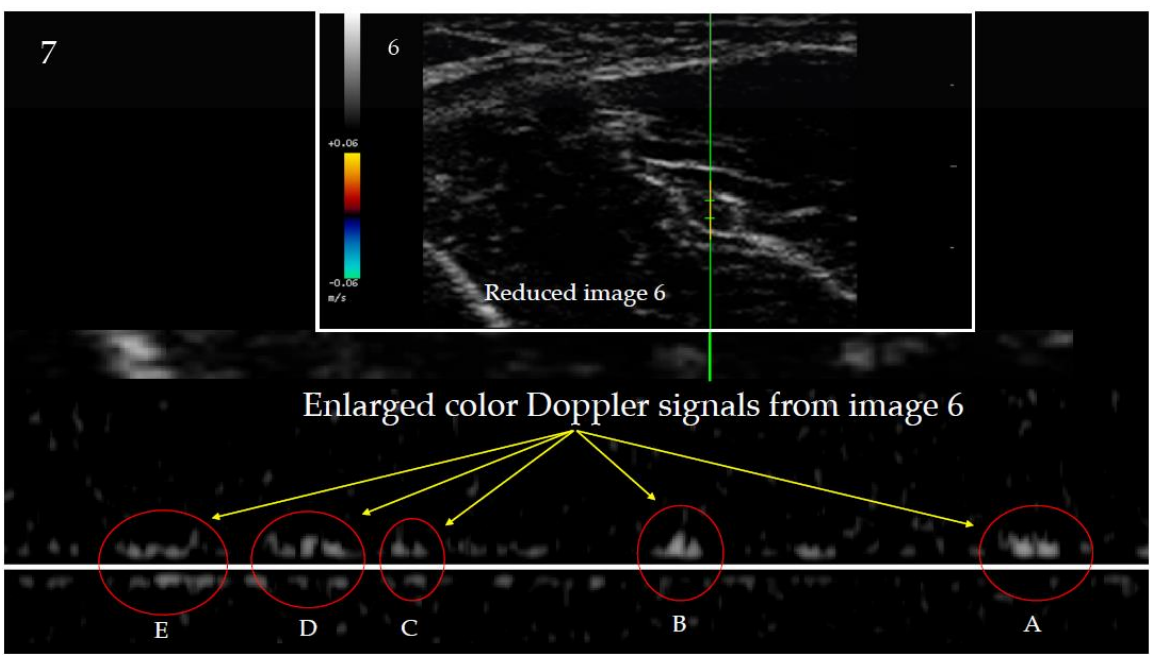

Fig 7: Ultrasonography of the median nerve, signals from color Doppler. Please notice, the lower panel is an enlarged section of the image from Fig. 6 that has been reduced in size 
Since it can be hypothesized that this effect on the mitochondria of cardiomyocytes is occurring also in cancer mitochondria, it can be deduced that restoration of mitochondria functionality might have been contributed to the clinical efficacy of these compounds (Ruggiero et al., 2014). We also published evidence supporting the hypothesis that the results observed in vitro and in vivo with VDR agonists complexed with amphipathic macromolecules and lipophilic compounds were due to the formation of supramolecular complexes of a glycosaminoglycan, chondroitin sulfate, with vitamin $\mathrm{D}_{3}$ and lipophilic molecules (Ruggiero et al., 2016). We recently described the molecular and functional characteristics of a supramolecular structure formed by microbial chondroitin sulfate, vitamin $\mathrm{D}_{3}$ and ultra-pure phosphatidylcholine (Ruggiero and Pacini, 2018a; 2018b) Below, we describe how each one of these components, has a role in restoring mitochondrial functionality and how these effects may occur at the quantum level.

Microbial chondroitin sulfate. It has been observed since 1969 that chondroitin sulfate dramatically reduces morbidity, i.e., coronary episodes, in patients with ischemic heart disease (Morrison, 1969). It has also been known since 1988 that chondroitin sulfate is responsible for the anti-cancer effects of cartilage preparations (Rosen et al., 1988; Prudden, 1985). It has been recently demonstrated that chondroitin sulfate improves mitochondrial activity in human cells by increasing the efficiency of metabolic processes, with particular reference to the generation of energy and reduction of ROS levels (Montell et al., 2015). Therefore, it can be deduced that the heart-supporting and anti-cancer effects of chondroitin sulfate are due to the restoration of mitochondrial energy generation and the decreased production of ROS. It is worth noticing that the observations quoted above refer to animal-derived chondroitin sulfate that is a heterogeneous mixture of high- and low-molecular-weight species (Ruggiero and Pacini, 2018a). The recent development of microbial chondroitin sulfate represents an evolution that may further improve the efficacy of chondroitin sulfate both in cardiology and oncology as well as in other conditions associated with mitochondrial dysfunction. Microbial chondroitin sulfate, at variance with its animal-derived counterpart, is characterized by over $99 \%$ of purity and it is homogeneous since it is constituted by low-molecularweight species. These characteristics are responsible for higher biological activity demonstrated by preclinical and clinical studies (Ruggiero and Pacini, 2018a). Such increased efficacy is not surprising as it is well known that low-molecular-weight glycosaminoglycans are much more effective than mixtures of high- and lowmolecular-weight species (Ruggiero and Pacini, 2018a). In addition, it is worth mentioning that ROS production in mitochondria is influenced by quantum phenomena, more precisely, coherent electron spin dynamics (Usselman et al., 2016).

Therefore, the effects of chondroitin sulfate on mitochondrial efficiency could be ascribed to the phenomena of enhanced coherence and entanglement that can be studied with the original ultrasonographic procedures described above. Chondroitin sulfate and in particular microbial chondroitin sulfate, carries plenty of quantum information associated with phenomena of entanglement between the electron clouds surrounding the highly charged glycosaminoglycan and the electron clouds of DNAs (Rieper et al., 2011), where the plural stands for mitochondrial and nuclear DNA. Thanks to its quantum properties, chondroitin sulfate could be considered the trait d'union that harmonizes information in the two cell compartments containing DNA. It is known that such a harmonization is essential for optimal health and longevity; alterations affecting the balance between genetic information contained in the nucleus and that in the mitochondrion leads to decreased efficiency of the electron transport chain that in turn leads to decreased energy generation and increased ROS production, two hallmarks of cancer and other conditions associated with mitochondrial dysfunction that include aging (Nunn et al., 2016). If mitochondria work as sensors balanced between the two realms of quantum and classical worlds where the relationship between the de-coherent macroscopic world and the coherent quantum world is responsible for mitochondrial function or dysfunction (Nunn et al., 2016), then the protocellular structures that we described in 2018 (Ruggiero and Pacini, 2018a) may act as quantum harmonizers for mitochondria (see below). It is also worth mentioning that microbial chondroitin sulfate is synthesized by nongenetically modified bacteria and, therefore, carries the entangled information originating from bacterial DNA. Genetic information in mitochondria shares many similarities with that of bacteria since "... genome evolution models now indicates that 97\% of modern mitochondrial protein domains as well their homologues in bacteria and archaea were present in the universal common ancestor of the modern tree of life" (Harisha and Kurlandb, 2017). This consideration further strengthens the concept that microbial chondroitin sulfate may act as a quantic trait d'union able to restore, in dysfunctional mitochondria, the primeval information at the basis of life on this planet as a sort of reboot that brings the mitochondrion to an unadulterated state.

Vitamin $D_{3}$. The inotropic effects of vitamin $D_{3}$ are well assessed (Witte et al., 2016) just like its anti-cancer effects that can be summarized as cancer prevention and adjuvant in cancer treatment (Chakraborti, 2011). As with chondroitin sulfate, co-existence of inotropic and anti-cancer effects leads to hypothesize that an influence 
on mitochondria is at the base of both phenomena. The actions of vitamin $\mathrm{D}_{3}$ at the level of mitochondria have been demonstrated in many cell types and it is well known that they are mediated by VDR. In particular, it was demonstrated that stimulation of VDR is associated with reduced formation of ROS in mitochondria (Ricca et al., 2018). These recent results are consistent with our hypothesis, first elaborated in 2013, that activation of VDR by a multi-molecular complex of VDR agonists, amphipathic macromolecules and lipophilic compounds was responsible for anti-cancer and inotropic effects (Pacini et al., 2013; Ruggiero et al., 2014; Thyer et al., 2013). In the design of the formula described (Ruggiero and Pacini, 2018a), vitamin $\mathrm{D}_{3}$ was chosen also because of its quantum properties that are complementary to those of chondroitin sulfate and trace their origins to the emergence of life on Earth. According to researchers from Lithuania and Norway "Life on Earth or elsewhere could have emerged in the form of prebiotic kernels and self-reproducing photoactive fatty acid micelles, which gradually evolved into nucleotide-containing micelles due to the enhanced ability of nucleotide-coupled sensitizer molecules to absorb visible light" (Tamulis and Grigalavicius, 2014). The Authors, Tamulis and Grigalavicius, describe how provitamin D molecules emerged from a quantum mechanical origin and favored harvesting of light in what they define prebiotic kernels that are structures similar to the protocellular structures we described in 2018 (Ruggiero and Pacini, 2018a). As far as the quantum properties of vitamin $\mathrm{D}_{3}$ are concerned, Tamulis and Grigalavicius state "Our discovered phenomenon of the quantum entanglement in the provitamin D prebiotic system enhance of photosynthesis in the system because simultaneously excite two prebiotic kernels in the system by appearance of two additional quantum entangled excited states. We can propose that quantum entanglement enhanced the emergence of photosynthetic prebiotic kernels and accelerated the evolution of photosynthetic life because of more absorbed light energy". (Tamulis and Grigalavicius, 2014). The similarity between the prebiotic kernels described by Tamulis and Grigalavicius (2014) and the protocellular structures made of chondroitin sulfate, phosphatidylcholine and vitamin $\mathrm{D}_{3}$ (Ruggiero and Pacini, 2018a) is further highlighted by the role played by fatty acids in establishing selfassembling micelles. In the formula described in 2018 (Ruggiero and Pacini, 2018a; 2018b), fatty acids are provided by ultrapure phosphatidylcholine following a molecular model that we described for the first time in 1985 (Vannucchi et al., 1985). The positively charged quaternary ammonium groups of the polar head of phosphatidylcholine bind, via electrostatic interactions, with the negatively charged carboxylic and sulfate groups of microbial chondroitin sulfate. From our paper of 1985 , it can be calculated that each molecule of microbial chondroitin sulfate is encircled by approximately 50 molecules of phosphatidylcholine that expose their hydrophilic tails constituted by fatty acids to the aqueous environment. Such a molecular arrangement induces the self-assembly of bilayers of phosphatidylcholine leading to cell membrane-like structures similar in principle to the prebiotic kernels described by Tamulis and Grigalavicius (2014) whose self-assembly, proceeding uniquely according to the laws of quantum physics, can be calculated and visualized by quantum mechanical time-dependent density functional theory. There is, however, a significant difference between the prebiotic kernels of Tamulis and Grigalavicius and the protocellular structures that we described in 2018 (Ruggiero and Pacini, 2018a; 2018b); the latter are homogeneous, single-phospholipid, bilayers that encircle low-molecular- weight microbial chondroitin sulfate instead of nucleic acids. We chose a glycosaminoglycan rather than a nucleic acid or a protein because the sequence of the former is repetitive and does not reveal biochemical or genetic information under the form of a sequence of four nitrogenous bases or twenty amino acids, thus avoiding recognition as "not-self" by the immune system.

However, as discussed above, microbial chondroitin sulfate carries plenty of information at the quantum level and this information is exploited to restore mitochondrial functionality by enhancing coherence thus increasing the generation of energy and decreasing the production of ROS. This noteworthy difference notwithstanding, there are no doubts that vitamin $\mathrm{D}_{3}$ plays an essential role both in the prebiotic kernels of Tamulis and Grigalavicius and in our protocellular structures acting as an intermediate evolved to harvest the energy of the sun at the chemical and quantum levels.

Phosphatidylcholine. It is well known that phosphatidylcholine is essential for mitochondrial functionality as it is one of the major constituents of the membrane of the organelle (Cigana Schenkel and Bakovic, 2014). It also known that phospholipids of the mitochondrial membrane are not only architectural components, but participate in the activity of respiratory proteins. Phosphatidylcholine is essential for protein transport into the inner membrane of mitochondria and into the matrix and depletion of phosphatidylcholine affects the integrity of the TIM23 translocase and precursor transfer to the TIM23 complex (Schuler et al., 2016). TIM23 complex is the translocase that transports precursor proteins with a cleavable pre-sequence into the inner membrane and the matrix. Phosphatidylcholine is also essential for the function of the Sorting and Assembly Machinery (SAM complex) of the outer membrane of the mitochondria (Schuler et al., 2016). In the approach described (Ruggiero 
and Pacini, 2018a), ultrapure phosphatidylcholine was used in order to exploit the properties of the well-described spontaneous association of circulating glycosaminoglycans and phosphatidylcholine (Vannucchi et al., 1985) and the resulting effects on negentropy as discussed (Ruggiero and Pacini, 2018a). In addition, phosphatidylcholine proves instrumental in determining the kinetics of absorption and intracellular delivery of the supramolecular complexes (Ruggiero and Pacini, 2018a). As far as the quantum properties of phosphatidylcholine are concerned, it is worth noticing that they are complementary to those of chondroitin sulfate and vitamin D3 and contribute to the overall efficiency of the formula described (Ruggiero and Pacini, 2018a). In addition to participating to the formation of the protocellular structures described above, phosphatidylcholine contributes to the process of geometry optimization for the supramolecular complexes constituted by the phospholipid, vitamin D3 and microbial chondroitin sulfate, thus introducing a further level of quantum information. In the example provided by Tamulis and Grigalavicius (2014), the prebiotic kernel is assembled with the tail of provitamin D oriented at 0 or 180 degrees to the opposite side of the tail of precursor fatty acid in order to optimize hydrophobic and electrostatic interactions. As mentioned before, according to Tamulis and Grigalavicius, such an assembly proceeds exclusively according to the laws of quantum physics. In the formula described (Ruggiero and Pacini, 2018a), these orientations are maintained as far as the interactions between vitamin $\mathrm{D}_{3}$ and phosphatidylcholine are concerned. However, there is a further layer of complexity due to electrostatic interactions between the $-\mathrm{OH}$ group of vitamin $\mathrm{D}_{3}$ and the negatively charged carboxylic and sulfate groups of microbial chondroitin sulfate that are not occupied by binding the polar head of phosphatidylcholine.

Because of these interactions, vitamin $\mathrm{D}_{3}$ lays on both sides of the membrane-like bilayer constituted by phosphatidylcholine self-assembled around microbial chondroitin sulfate. Physical interaction between the electron clouds of the three molecules gives rise to a complex form of entanglement that is probably responsible for sustaining augmented coherence, ultimately contributing to the observed biological effects of the formula.

From the functional point of view, the formula described by Ruggiero and Pacini is based on three natural compounds each one endowed with the ability to restore mitochondrial physiology both at the macroscopic and the quantum levels. The efficacy of each compound is enhanced by the formation of non-covalent bonds with the other two so to form supramolecular complexes able to target different aspects of mitochondrial metabolism simultaneously, at the same time exhibiting an augmented state of quantum entanglement and coherence. These effects on mitochondrial functionality are probably crucial in determining the responses observed in autism and cancer (Antonucci et al., 2018; 2019a; 2019b). It is well established that autism is associated with mitochondrial dysfunction and defective energy production (Siddiqui et al., 2016) and it is therefore not surprising that an approach based on restoring mitochondrial functionality may help in this condition. As far as the case described by Antonucci et al. (2019b) is concerned, it is worth considering that this neoplasia is among those that accumulate iron, a metal essential to promote cancer cell growth and resistance to chemotherapy. High levels of iron in the cytosol react with ROS produced by defective mitochondria in cancer cells and this leads to further oxidative damage (Zhan et al., 2017). It is therefore plausible that the encouraging results reported by (Antonucci et al., 2019b) may be ascribed also to the restoration of mitochondrial functionality associated with detoxification of metals as also described in (Blythe et al., 2017).

\section{Conclusion}

The procedures described for the first time in this study are consistent with the novel applications of ultrasonography in diagnostics and therapy that we first proposed with the Ruggiero-Klinghardt Protocol in 2017 (Antonucci et al., 2018; Klinghardt and Ruggiero, 2017). Color Doppler ultrasonography of the radial artery to measure IRVT and study of Doppler signals arising from within axons of peripheral nerves, will prove instrumental in preventively assessing the efficacy of approaches aimed at restoring mitochondrial functionality in all conditions associated with their dysfunction, from cancer to autism, neurodegenerative diseases, heart conditions, myalgic encephalomyelitis/chronic fatigue syndrome and aging.

\section{Acknowledgement}

The Authors wish to thank imuno Corporation, Vanuatu, for development of the formula described by Ruggiero and Pacini and for inspiring discussion.

\section{Authors' Contribution}

Stefania Pacini, MD, PhD and Marco Ruggiero, MD, $\mathrm{PhD}$, contributed equally to this paper.

\section{Disclosures}

Marco Ruggiero is the founder of Silver Spring Sagl, a Swiss company producing probiotics and other supplements that are described in some of the references. Stefania Pacini works as for Silver Spring Sagl. No product of Silver Spring Sagl is mentioned in the present study. Marco Ruggiero and Stefania Pacini also consult for other companies and have 
invented/developed several supplements and protocols that are described in some of the references. Marco Ruggiero is member of the Editorial Board of the American Journal of Immunology and is waived from the Article Processing fee for this contribution; he receives no remuneration for his editorial work.

\section{Advisory}

No information in this paper is intended or implied to be a substitute for professional medical advice, diagnosis or treatment.

\section{Ethics}

This article is original and contains material that has not been published in any journal. All images are from the professional archive of Marco Ruggiero, MD, radiologist, do not refer to any patient or member of the public and are presented solely as examples of applications of ultrasonography.

\section{References}

Antonucci, N., D. Klinghardt, S. Pacini and M. Marco Ruggiero, 2018. Tailoring the ruggiero-klinghardt protocol to immunotherapy of autism. Am. J. Immunol., 14: 34-41.

DOI: 10.3844/ajisp.2018.34.41

Antonucci, N., S. Pacini and M. Ruggiero, 2019. Clinical experience of integrative autism treatment with a novel type of immunotherapy. Madridge J. Vaccines, 3: 71-76. DOI: 10.18689/mjv-1000116

Antonucci, N., S. Pacini and M. Ruggiero, 2019. Use of an extremely biodiverse probiotic and a supplement based on microbial chondroitin sulfate is associated with a significant decrease of serum free kappa light chains as well as a trend toward normalization of kappa/lambda ratio and of plasma cell bone marrow infiltration in a case of multiple myeloma. Am. J. Immunol., 15: 5-9. DOI: 10.3844/ajisp.2019.5.9

Blythe, J., R. Marco and S. Pacini, 2017. Case Report: Intermittent fasting and probiotic yogurt consumption are associated with reduction of serum alpha-n-acetylgalactosaminidase and increased urinary excretion of lipophilic toxicants. Madridge J. Immunol., 1: 23-27. DOI: 10.18689/mjim-1000107

Brecker, S.J.D., C.H. Lee and D.G. Gibson, 1992. Relation of left ventricular isovolumic relaxation time and incoordination to transmitral Doppler filling patterns. Heart, 68: 567-573.

DOI: $10.1136 /$ hrt.68.12.567

Chakraborti, C.K., 2011. Vitamin D as a promising anticancer agent. Indian J. Pharmacol., 43: 113-120. DOI: $10.4103 / 0253-7613.77335$
Chang, J.C., H.S. Chang, Y.C. Wu, W.L. Cheng and T.T. Lin et al., 2019. Mitochondrial transplantation regulates antitumour activity, chemoresistance and mitochondrial dynamics in breast cancer. J. Exp. Clin. Cancer Res., 38: 30-30.

DOI: 10.1186/s13046-019-1028-z

Cigana Schenkel, L. and M. Bakovic, 2014. Formation and regulation of mitochondrial membranes. Int. J. Cell Biol., 6: 709828-709828. DOI: $10.1155 / 2014 / 709828$

Guo, R., J. Gu, S. Zong, M. Wu and M. Yang, 2018. Structure and mechanism of mitochondrial electron transport chain. Biomed. J., 41: 9-20.

DOI: 10.1016/j.bj.2017.12.001

Hameroff, S. and R. Penrose, 2014. Consciousness in the universe: A review of the 'Orch OR' theory. Phys. Life Rev., 11: 39-78.

DOI: $10.1016 /$ j.plrev.2013.08.002

Harisha, A. and C.G. Kurlandb, 2017. Mitochondria are not captive bacteria. J. Theor. Biol., 434: 88-98. DOI: 10.1016/j.jtbi.2017.07.011

Hirpara, A., M. Bloomfield and P. Duesberg, 2018. Speciation theory of carcinogenesis explains karyotypic individuality and long latencies of cancers. Genes, 9: 402-402. DOI: 10.3390/genes908040

Klinghardt, D. and M. Ruggiero, 2017. The RuggieroKlinghardt (RK) protocol for the diagnosis and treatment of chronic conditions with particular focus on lyme disease. Am. J. Immunol., 13: 114-126. DOI: 10.3844/ajisp.2017.114.126

Luckey, T.D., 2008. Atomic bomb health benefits. Dose Respon., 6: 369-382.

DOI: 10.2203/dose-response.08-009.Luckey

McCully, J.D., S. Levitsky, P.J. Del Nido and D.B. Cowan, 2016. Mitochondrial transplantation for therapeutic use. Clin. Trans. Med., 5: 16-16. DOI: 10.1186/s40169-016-0095-4

Montell, E., M. López-Armada, J. Vergés, C. RuizRomero and F.J. Blanco et al., 2015. Mitochondria: A new chondroitin sulphate therapeutic target for osteoarthritis. Clin. Thera., 8: e120-e121.

DOI: $10.1016 /$ j.clinthera.2015.05.345

Morrison, L.M., 1969. Response of ischemic heart disease to chondroitin sulfate-a. J. Am. Geriatr. Society, 17: 913-923. DOI: 10.1111/j.1532-5415.1969.tb02328.x

Nunn, A.V., G.W. Guy and J.D. Bell, 2016. The quantum mitochondrion and optimal health. Biochem. Soc. Trans., 44: 1101-1110. DOI: $10.1042 / B S T 20160096$

Pacini, S. and M. Ruggiero, 2019. Phage composition of a fermented milk and colostrum product assessed by microbiome array; putative role of open reading frames. 
Pacini, S., G. Morucci, J.J. Branca, S. Aterini and M. Amato et al., 2013. Effects of vitamin D3 and paricalcitol on immature cardiomyocytes: A novel role for vitamin D analogs in the prevention of cardiovascular diseases. Nutrients, 5: 2076-2092. DOI: $10.3390 /$ nu5062076

Prudden, J.F., 1985. The treatment of human cancer with agents prepared from bovine cartilage. J. Biol. Response Mod., 4: 551-84. PMID: 4087031

Ricca, C., A. Aillon, L. Bergandi, D. Alotto and C. Castagnoli et al., 2018. Vitamin D receptor is necessary for mitochondrial function and cell healthint. J. Mol. Sci., 19:1672-1672.

DOI: $10.3390 /$ ijms 19061672

Rieper, E., J. Anders and V. Vedral, 2010. Quantum entanglement between the electron clouds of nucleic acids in DNA. arXiv: 1006.4053 [quant-ph]. https://arxiv.org/pdf/1006.4053.pdf

Rosen, J., W.T. Sherman, J.F. Prudden and G.J. Thorbecke, 1988. Immunoregulatory effects of catrix. J. Biol. Response Mod., 7: 498-512. PMID: 2846789

Ruggiero, M. and S. Pacini, 2018. Rationale for the design of a novel tool for immunotherapy based on an emulsion of glycosaminoglycan. Integr. Cancer Sci. Therap. DOI: 10.15761/ICST.1000285

Ruggiero, M. and S.A. Pacini, 2018. Novel potential adjuvant for cancer vaccines. Madridge J. Vaccines. 2: 57-61. DOI: $10.18689 / \mathrm{mjv}-1000112$

Ruggiero, M., E. Ward, R. Smith, J.J. Branca and D. Noakes et al., 2014. Oleic acid, deglycosylated vitamin d-binding protein, nitric oxide: A molecular triad made lethal to cancer. Antic. Res., 34: 3569-3578. PMID: 24982371

Ruggiero, M., H. Reinwald and S. Pacini, 2016. Is chondroitin sulfate responsible for the biological effects attributed to the GC protein-derived Macrophage Activating Factor (GcMAF)? Med. Hypotheses, 94: 126-131.

DOI: 10.1016/j.mehy.2016.07.012

Sajic, M., V. Mastrolia, C.Y. Lee, D. Trigo and M. Sadeghian et al., 2013. Impulse conduction increases mitochondrial transport in adult mammalian peripheral nerves in vivo. PLoS Biol., 11: e1001754-e1001754. DOI: 10.1371/journal.pbio.1001754

Schuler, M.H., F. Di Bartolomeo, C.U. Mårtensson, G. Daum and T. Becker, 2016. Phosphatidylcholine affects inner membrane protein translocases of mitochondria. J. Biol. Chem., 291: 18718-18729. DOI: $10.1074 /$ jbc.M116.722694

Seyfried, T., 2015. Cancer as a mitochondrial metabolic disease. Front. Cell Dev. Biol., 3: 43-43.

DOI: $10.3389 /$ fcell.2015.00043

Shibamoto, Y. and H. Nakamura, 2018. Overview of biological, epidemiological and clinical evidence of radiation hormesis. Int. J. Mol. Sci., 19: 2387-2387. DOI: 10.3390/ijms19082387
Siddiqui, M.F., C. Elwell and M.H. Johnson, 2016. Mitochondrial dysfunction in autism spectrum disorders. Autism Open Access, 6: 1000190-000190. DOI: $10.4172 / 2165-7890.1000190$

Tamulis, A. and M. Grigalavicius, 2014. Quantum entanglement in photoactive prebiotic systems. Syst. Synth. Biol., 8: 117-140.

DOI: $10.1007 / \mathrm{s} 11693-014-9138-6$

Tapia, P.C., 2006. Sublethal mitochondrial stress with an attendant stoichiometric augmentation of reactive oxygen species may precipitate many of the beneficial alterations in cellular physiology produced by caloric restriction, intermittent fasting, exercise and dietary phytonutrients: "Mitohormesis" for health and vitality. Med. Hypothe, 66: 832-43. DOI: 10.1016/j.mehy.2005.09.009

Thyer, L., E. Ward, R. Smith, M.G. Fiore and S. Magherini et al., 2013. A novel role for a major component of the vitamin D axis: Vitamin D binding protein-derived macrophage activating factor induces human breast cancer cell apoptosis through stimulation of macrophages. Nutrients. 5: 2577-2589. DOI: 10.3390/nu5072577

Usselman, R.J., C. Chavarriaga, P.R. Castello, M. Procopio and T. Ritz et al., 2016. The quantum biology of reactive oxygen species partitioning impacts cellular bioenergetics. Sci. Repo., 6: 38543-38543. DOI: 10.1038/srep38543

Vannucchi, S., M. Ruggiero and V. Chiarugi, 1985. Complexing of heparin with phosphatidylcholine. A possible supramolecular assembly of plasma heparin. Biochem. J., 227: 57-65.

DOI: $10.1042 / \mathrm{bj} 2270057$

Warburg, O., 1956. On the origin of cancer cells. Sci., 123: 309-314. DOI: 10.1126/science.123.3191.309

Witte, K.K., R. Byrom, J. Gierula, M.F. Paton and H.A. Jamil et al., 2016. Effects of vitamin D on cardiac function in patients with chronic HF: The vindicate study. J. Am. College Cardio., 67: 2593-603.

DOI: $10.1016 /$ j.jacc.2016.03.508

Woods, D.C. and J.L. Tilly, 2015. Autologous Germline Mitochondrial Energy Transfer (AUGMENT) in human assisted reproduction. Semin. Reprod. Med., 3: 410-421. DOI: 10.1055/s-0035- 1567826

Zhan, X., W. Yu and R. Franqui-Machin, 2017. Alteration of mitochondrial biogenesis promotes disease progression in multiple myeloma. Oncotarget, 8: 111213-111224. DOI: 10.18632 /oncotarget.22740 